\title{
Protein Turnover
}

National Cancer Institute

\section{Source}

National Cancer Institute. Protein Turnover. NCI Thesaurus. Code C21064.

The normal "downside" of protein steady state metabolism in cells, involving conjug ation, transport, oxidation, and proteolysis; not to be confuse with proteolysis, which applies only to peptide bond hydrolysis. 